Delayed cranioplasty-Titanium strips may sometimes be used instead of a preformed plate. If the fracture involves the frontal air inuses or mastoid air cells, however, a plate is usually preferables because it may be fixed to the skull with screws that do not penetrate areas of potential infection.

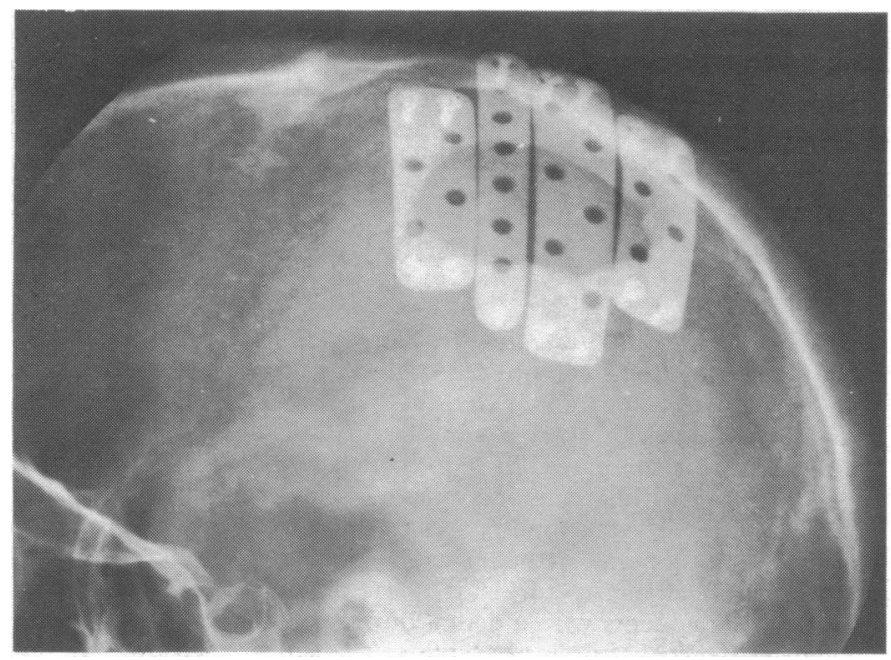

FIG 3-Titanium strips of various widths and lengths used for cranioplasty after debridement of missile wound.

\section{Comment}

Titanium-strip cranioplasty is a simple technique requiring only a dental drill, orthodontic pliers, and a basic head-injury craniotomy set. Titanium is radiolucent, which is important should contrast radiography become necessary. Its low specific gravity means that when used for large defects the patient will not be conscious of its weight. Titanium strips curved to the shape of the skull offer much greater resistance than uncontoured strips to high-impact loading. With one or two screws at each end the strips provide a strong protective cover.

This onlay technique has two chief advantages. Firstly, it obviates the need to dissect the dura mater at the margin of the defect, with consequent risk of a cerebrospinal fluid fistula or of brain damage from dissection on the undersurface of the bone. Secondly, as the strips overlie the skull for $1 \mathrm{~cm}$ or so each side of the defect, the resistance offered to further impact injury is greater than that from an inlay technique.

\section{References}

1 Simpson, D, Fournal of Neurosurgery, 1965, 22, 292.

2 Gordon, D S, and Blair, G A S, British Medical fournal, 1974, 2, 478.

3 Simpson, D, personal communication, 1976.

4 Meachin, G, and Williams, D F, fournal of Biomedical Materials Research, 1973, 7, 555.

\title{
Treatment of candidal urinary tract infection with nifuratel
}

\author{
R N GRÜNEBERG，ANNE LEAKEY
}

British Medical fournal, 1976, 2, 908-910

\section{Summary}

Three patients with candidal urinary tract infections were successfully treated with oral nifuratel, a nitrofuran antimicrobial agent active against yeasts and Trichomonas as well as urinary bacterial pathogens. The recommended dose is $\mathbf{4 0 0} \mathrm{mg}$ thrice daily for a week. No side effects that could be attributed to the treatment were noted. Minimum inhibitory concentration determinations for nifuratel against Candida strains of five species showed that 48 out of 59 organisms were inhibited by $50 \mathrm{mg} / 1$ or less, the three strains of Candida species eliminated from our treated patients having MICs of nifuratel in the range of $10-50 \mathrm{mg} / 1$.

\section{Introduction}

Candida species only rarely cause urinary tract infections, usually after urethral catheterisation in patients with urinary tract abnormalities who have received broad-spectrum antibiotics. The treatment of candidal urinary tract infection is difficult, partly because only a few antimicrobial agents are excreted in the urine and are active against yeasts. ${ }^{1}$

Nystatin is not well absorbed from the intestine and is too toxic for parenteral use, and amphotericin B, which may be used parenterally, is apt to cause serious side effects. The treat-

Department of Microbiology, University College Hospital, London WC1

R N GRÜNEBERG, MD, MRCPATH, consultant microbiologist ANNE LEAKEY, BSC, research technician ment of urinary tract infection caused by $C$ tropicalis with polymyxin has been described, ${ }^{2}$ but this antibiotic is not usually applicable to infections with other Candida species. Recently flucytosine has been used for these infections, but it is limited in its application because some strains of Candida $^{3}$ are resistant to it and because the dosage has to be modified in those with renal impairment.

Nifuratel is a nitrofuran which is used to treat vaginal infections with Candida or Trichomonas vaginalis, the recommended application being a combination of vaginal pessaries and tablets. Since oral nifuratel is excreted in the urine and the drug was presumed to be active against yeasts it seemed appropriate to try its effect on candidal urinary tract infections.

We tested Candida strains for sensitivity to nifuratel in vitro, and report here three cases of candidal urinary tract infection treated with nifuratel.

\section{Case 1}

A 77-year-old man fell and fractured his third lumbar vertebra. No neurological abnormality was detected and he was treated with bed rest. Two days after his fall he developed a chest infection, which was treated successfully with ampicillin given for two weeks. Three urine specimens examined during treatment were normal.

Nine days after the accident he developed acute urinary retention and a urethral catheter was passed. The catheterisation was difficult and the presence of urethral stricture was suspected, especially since he gave a history of gonorrhoea in the remote past. Closed drainage of the bladder through an indwelling catheter was started. Eight days later his urine grew more than $10^{5} \mathrm{C}$ stellatoidea $/ \mathrm{ml}$, a finding confirmed on five subsequent cultures. A course of nifuratel tablets, $200 \mathrm{mg}$ three times daily for seven days was given. The catheter was removed on the fourth day of treatment. Urine cultures at the end of the course of treatment again yielded more than $10^{5} \mathrm{C}$ stellatoidea $/ \mathrm{ml}$. A second course of treatment with nifuratel, this time $400 \mathrm{mg}$ three times daily for seven days, was given. Further urine examination after this course showed no evidence of infection. Two months later his urine remained clear. 
Case 2

A 58-year-old woman who was taking prednisone for rheumatoid arthritis was admitted to hospital with a vesicocolic fistula. At operation this was found to be caused by diverticulitis, and partial cystectomy and partial colectomy were performed. An indwelling catheter was left in position to drain the bladder. A pre-existing urinary infection with a sensitive Escherichia coli was treated with ampicillin and eradicated, to be replaced three days after the operation by $C$ tropicalis. Six urine samples collected over the next four days all yielded $>10^{5} / \mathrm{ml}$ of this organism. Treatment with nifuratel $200 \mathrm{mg}$ three times daily for seven days was started when the urethral catheter was removed, some seven days after operation. Follow-up examinations of urine samples over the next six weeks showed no evidence of infection.

\section{Case 3}

A 66-year-old man was admitted to hospital with hemiplegia but after one month developed bowel symptoms and was then found to have a carcinoma of the rectum, which was removed by abdominoperineal resection. Postoperatively the bladder was drained by an indwelling catheter. Many courses of antibacterial prophylaxis and treatment for chest infection were given, including ampicillin, penicillin G, streptomycin, co-trimoxazole, and tetracycline. One month after operation $C$ albicans was grown from a catheter urine sample and $>10^{5} / \mathrm{ml}$ of this organism was found in eight more specimens over the next six weeks. Nifuratel $400 \mathrm{mg}$ three times daily was then given for seven days, but the $C$ albicans persisted. A second similar course of nifuratel was given, which cleared the $C$ albicans, but the urine then reinfected with another yeast $-C$ stellatoidea. That this was a reinfection rather than a relapse was suggested not only by the different fermentative characteristics of the two yeasts but also by measurement, using a tube dilution technique, ${ }^{5}$ of the minimum inhibitory concentration (MIC) of nifuratel for the two yeasts, though the change in sensitivity might have been induced by treatment. The MIC of nifuratel for the $C$ albicans strain was $10 \mathrm{mg} / \mathrm{l}$ while that of the $C$ stellatoidea strain was $50 \mathrm{mg} / \mathrm{l}$. Further attempts to treat the urinary infection with nifuratel were not thought to be justified and the patient retained his candidal urinary tract infection until his death from carcinomatosis some months later.

\section{Methods}

Fifty-nine strains of Candida were isolated from a variety of clinical specimens from various sources in hospitals and general practices and were identified by standard fermentation techniques.

The solubility of nifuratel in water is low, so large amounts of an organic solvent, dimethylformamide, were used to prevent the nifuratel from precipitating out when added to broth. Since dimethylformamide is itself antimicrobial the maximum final concentration of the solvent in broth was adjusted to $2 \cdot 5^{\circ}$, , which we have shown not to affect the growth of micro-organisms. ${ }^{5}$ This strength of solvent limited the maximum final concentration of nifuratel in the test system to $50 \mathrm{mg} / \mathrm{l}$. A stock solution of $200 \mathrm{mg} / \mathrm{l}$ in dimethylformamide was added to Oxoid Nutrient Broth No 2 (sterilised and adjusted to $\mathrm{pH} 7 \cdot 0$ ) and appropriately diluted to give a final range of concentrations of nifuratel in broth of $50,25,10,5,2 \cdot 5$, and $1 \mathrm{mg} / 1.1-\mathrm{ml}$ volumes of these solutions were distributed in sterile round-bottomed glass tubes $12.5 \mathrm{~mm}$ in diameter. Two additional tubes were set up for each strain tested, one containing nutrient broth only and the other containing $2.5 \%$ dimethylformamide in nutrient broth.

Overnight broth cultures of the Candida strains were diluted 1/100 in normal saline, and one drop (containing 1-9 $\times 10^{5}$ colony-forming units) was added to each tube. One drop was also spread on a Sabouraud's agar plate to check organism density and purity. The tubes were inspected after overnight incubation at $37^{\circ} \mathrm{C}$, the MIC being taken as the lowest concentration of nifuratel in which there was no turbidity.

\section{Results}

The results of determining the MIC of nifuratel for 59 strains of Candida are shown in the table. All strains grew satisfactorily in the nutrient broth alone and also in nutrient broth containing $2.5 \%$ dimethylformamide, the highest concentration present in any tube. MICs ranged from less than 1 to over $50 \mathrm{mg} / \mathrm{l}$, with 48 of the 59 strains tested having MICs of nifuratel of $50 \mathrm{mg} / \mathrm{l}$ or less.
Minimum inhibitory concentrations of nifuratel for Candida spp

\begin{tabular}{|c|c|c|c|c|c|c|c|c|c|c|}
\hline \multirow{2}{*}{\multicolumn{2}{|c|}{ Organism }} & \multirow{2}{*}{$\begin{array}{l}\text { No of } \\
\text { strains } \\
\text { tested }\end{array}$} & \multicolumn{8}{|c|}{$\begin{array}{l}\text { No of strains inhibited by various } \\
\text { concentrations of nifuratel (mg/l) }\end{array}$} \\
\hline & & & $>50$ & 50 & 25 & 10 & 5 & 2.5 & 1 & $<1$ \\
\hline $\begin{array}{l}C \text { albicans } \\
C \text { stellatoidea } \\
C \text { parakrusei } \\
C \text { krusei } \\
C \text { tropicalis }\end{array}$ & $\begin{array}{l}\ldots \\
\cdots \\
\cdots \\
\cdots\end{array}$ & $\begin{array}{r}43 \\
9 \\
3 \\
2 \\
2\end{array}$ & $\begin{array}{l}9 \\
2\end{array}$ & $\begin{array}{r}24 \\
4 \\
2 \\
2 \\
1\end{array}$ & $\begin{array}{l}7 \\
1\end{array}$ & $\begin{array}{l}3 \\
1\end{array}$ & & 1 & & 1 \\
\hline Total & & 59 & 11 & 33 & 9 & 4 & & 1 & & 1 \\
\hline
\end{tabular}

\section{Discussion}

Candidal urinary tract infection is rare, occurring much more often in hospitals than elsewhere. We isolated Candida spp in 39 out of 3157 cases of urinary tract infection in hospital and in six out of 2532 cases in general practice that were diagnosed in the St Pancras Hospital laboratory in 1971-5. All three of the patients described had had indwelling urethral catheters, two had some pre-existing urinary tract abnormality, and all had received broad-spectrum antibiotics, notably ampicillin. The treatment of any urinary tract infection in such unpromising circumstances would be difficult. The basis for claiming that these patients were infected with Candida was the usual one of multiple urine cultures yielding more than $10^{5}$ organisms $/ \mathrm{ml}$ of urine. Suprapubic aspirations of bladder urine were not performed.

The nitrofurans are a group of synthetic antimicrobial drugs, the best known being nitrofurantoin, which is widely used for treating bacterial urinary tract infection. Nifuratel (Magmilor) has the same antibacterial spectrum of activity as nitrofurantoin and seems to be somewhat more active weight for weight. ${ }^{5}$ Nifuratel has not been much used for treating urinary tract infection, but it has been widely prescribed for treating trichomonal infections and candidal infections of the vagina. The usual method of administration for these vaginal conditions has been a combination of vaginal pessaries and tablets. Since the nitrofurans are excreted in the urine and are, presumably, active against Candida spp the use of nifuratel tablets seemed to offer a possible method of treating candidal urinary tract infection.

There is a lack of reliable information on the pharmacokinetics of nifuratel. Assay techniques with nitrofurans are notoriously difficult: thus one study ${ }^{6}$ showed that antibacterial concentrations of nifuratel are almost never achieved in the urine-a finding that does not accord well with the high cure rates of bacterial urinary tract infection achieved by nifuratel treatment. ${ }^{7-9} \mathrm{We}$ have shown that the urine of patients with candidal urinary tract infection is qualitatively inhibitory to their urinary pathogens during nifuratel treatment, but we have no quantitative data. The in-vitro activity of nifuratel, like that of nitrofurantoin, is greatly affected by $\mathrm{pH}$, being much more active in acid than in alkaline media. ${ }^{5}$

The results of treatment in our three patients were varied. The $C$ stellatoidea infection in case 1 failed to respond to $200 \mathrm{mg}$ of nifuratel three times daily but was cleared by $400 \mathrm{mg}$ three times daily for seven days. The $C$ tropicalis infection in case 2 was eradicated by the lower dose of nifuratel for seven days. The third patient had a $C$ albicans infection that was not affected by the first course of high-dose nifuratel. A second highdose course eliminated the $C$ albicans infection but caused reinfection with another and more resistant yeast, $C$ stellatoidea. From the academic standpoint the drug must be credited with eradicating the infecting organism, though the patient benefited little from this. In general it seems that when nifuratel is used to treat candidal urinary tract infections it should be given in high dosage-that is, $400 \mathrm{mg}$ thrice daily for a week. No side effects were noted during the five courses of nifuratel given to these three patients.

The results of MIC determinations for nifuratel against Candida spp indicate that 48 out of 59 strains tested had MICs of $50 \mathrm{mg} / \mathrm{l}$ or less. There is no satisfactory information on the 
concentrations of nifuratel obtained in the urine of treated subjects, so that the significance of our MIC findings for clinical practice is difficult to assess. The MICs of nifuratel of the three strains that were successfully eradicated from our patients were: $10 \mathrm{mg} / 1$ (C albicans), $25 \mathrm{mg} / 1$ ( $C$ tropicalis), and $50 \mathrm{mg} / 1$ ( $C$ stellatoidea). Most of the strains tested by us have had MICs in this range.

It seems reasonable to consider nifuratel as a possible choice for treating candidal urinary tract infection when the infection is thought to merit chemotherapy.

We are grateful to our colleagues, Mr D R Davies, Mr E Kirwan, and Professor A N Exton-Smith, for permission to treat patients under their care. Dr E Letley of Calmic Ltd and Dr A Salter of the Wellcome Foundation kindly supplied nifuratel tablets and powder. We are grateful for the meticulous care in specimen collection and follow-up shown by the nurses tending these patients.

\section{References}

1 Speller, D C E, fournal of Antimicrobial Chemotherapy, 1975, 1, 253.

${ }^{2}$ Nicholls, M W N, fournal of Medical Microbiology, 1970, 3, 529.

3 Speller, D C E, and Davies, M G, Fournal of Medical Microbiology, 1973, 6, 315.

4 Schönebeck, J, et al, Chemotherapy, 1973, 18, 321.

5 Grüneberg, R N, in Diagnosis and Chemotherapy of Urogenital Infections, Ed F Gasparri, p 109. Florence, Edizione Mediche P Periti, 1972.

6 McGeachie, J, Robinson, G, and Black, D, fournal of Clinical Pathology, $1972,25,447$.

7 Tynan, A P, Macis, F R, and Ward-McQuaid, J N, British fournal of Urology, 1969, 41, 271.

${ }^{8}$ Gower, P E, Clinical Trials fournal, 1970, 7, 389.

9 Brumfitt, W, in Diagnosis and Chemotherapy of Urogenital Infections, ed F Gasparri, p 411. Florence, Edizione Mediche P Periti, 1972.

\title{
Preoperative prediction of postoperative deep vein thrombosis
}

\author{
J K CLAYTON, J A ANDERSON, G P MCNICOL
}

British Medical fournal, 1976, 2, 910-912

\section{Summary}

A range of clinical data was obtained from 124 patients about to undergo operation and several coagulation tests were performed. No patient received prophylaxis for deep vein thrombosis, and isotopic scanning after operation showed that 20 patients had developed thrombosis. A simple prognostic index for predicting which patients would develop postoperative deep vein thrombosis was constructed using the clinical and coagulation data obtained before operation. The five variables with the best predictive power-euglobulin lysis time, age, presence of varicose veins, fibrin related antigen, and percentage overweight-produced an equation that identified $95 \%$ of those who developed deep vein thrombosis and misallocated only $28 \%$ of those who did not develop thrombosis. In view of the complications that low-dose heparin and dextran can cause, giving prophylaxis to under a third of the patients who will not develop deep vein thrombosis is clearly better than giving it to all.

\section{Introduction}

Recently impressive evidence has accumulated that the incidence of postoperative deep vein thrombosis can be reduced by prophylactic measures, particularly low-dose heparin and perioperative dextran 70.12 Both low-dose heparin and dextran may, however, significantly increase bleeding problems at the

Departments of Obstetrics and Gynaecology, Community Medicine and General Practice, and Medicine, University of Leeds, Leeds LS1 3EX

J K CLAYTON, MRCOG, senior registrar in obstetrics and gynaecology

$\mathrm{J}$ A ANDERSON, MA, DPHIL, (now reader in statistics, University of Newcastle upon Tyne)

G P MCNICOL, MD, PHD, professor of medicine time of surgery, ${ }^{34}$ and our object was to try to identify before operation those patients particularly at risk of deep vein thrombosis in the hope that in future it would be unnecessary to give prophylactic treatment to patients in a low-risk group.

\section{Patients and methods}

One hundred and twenty-four patients undergoing major gynaecological surgery, both abdominal and vaginal, were investigated. The following clinical data were obtained: age; weight; height; length of stay in hospital before operation; preoperative haemoglobin level; smoking habits; presence of varicose veins on clinical examination; history of venous thromboembolic disease; the nature of the operation; and whether surgery was for benign or malignant disease.

Laboratory methods-Platelets were measured on a Coulter thrombocounter. Platelet factor III release was measured by the method of Spaet and Cintron, ${ }^{5}$ fibrinogen by the method of Ratnoff and Menzie, ${ }^{6}$ antithrombin III by the method of Howie et al, ${ }^{7}$ factor VIII by a onestage method of Breckenridge and Ratnoff, ${ }^{8}$ euglobulin lysis time by the method of Nilsson and Olow, ${ }^{9}$ serum fibrin related antigen (FR antigen) by the method of Merskey et $a l,{ }^{10}$ plasminogen by the method of Alkjaersig et al, ${ }^{11}$ antiplasmin by the method of Howie et $a l,,^{12}$ activated partial thromboplastin time by the method of Langdell et al, ${ }^{13}$ and thrombin time by the method of Eichelberger. ${ }^{14}$ Platelet aggregation was measured to adenosine diphosphate (ADP) $1 \mu \mathrm{mol} / \mathrm{l}, 4 \mu \mathrm{mol} / \mathrm{l}$, and $10 \mu \mathrm{mol} / 1$; to collagen; and to adrenaline by the aggregometer method of Born and Cross. ${ }^{15}$

Diagnosis of deep vein thrombosis-All patients underwent preoperative isotropic scanning of the legs using the ${ }^{125}$ I-fibrinogen technique with the criteria of Kakkar. ${ }^{16}$ Routine postoperative scanning was performed on the first, third, and sixth days unless a significantly high count was obtained when daily scanning was started. None of the patients had received specific prophylaxis for deep vein thrombosis.

\section{Results and comment}

No patients had evidence of deep vein thrombosis before surgery. After operation 20 of the 124 patients developed isotopic evidence of deep vein thrombosis, which was confirmed by ascending venography in all of the 18 in whom this procedure was performed.

Clinical and coagulation data on the 104 patients without deep vein thrombosis after operation and the 20 with deep vein thrombosis are shown in the table. 